\title{
INFLUÊNCIA DA METODOLOGIA DE ESTIMAÇÃO SOBRE OS PARÂMETROS CINÉTICOS E O PROJETO DE REATORES CONTÍNUOS
}

\author{
G. S. S. SARCINELLI ${ }^{1}$, L. G. R. MIRANDA ${ }^{1}$, J. C. S. DUTRA ${ }^{1}$, F. T. VIEIRA ${ }^{1}$ \\ ${ }^{1}$ LAMCES - Laboratório de Métodos Computacionais, Controle e Estimação \\ Universidade Federal do Espírito Santo - Engenharia Química (campus Alegre) \\ e-mail para contato: gabriela.sarcinelli@gmail.com
}

\begin{abstract}
RESUMO - Os procedimentos de estimação de parâmetros tanto tornam possíveis as interpretações qualitativas e quantitativas de dados experimentais como a simulação e o projeto de processos. Para a cinética química, estas ferramentas são importantes na predição da lei de velocidade de uma reação. Neste processo, a análise cinética é feita a partir de dados experimentais coletados durante a evolução de uma reação química, através da medição da concentração em função do tempo, e do uso de métodos de análise, por onde será possível estimar os parâmetros cinéticos desta reação, que são a ordem de reação e a velocidade específica de reação. Tradicionalmente, as técnicas de estimação de parâmetros cinéticos envolvem a manipulação do modelo, de forma que estes tornem-se lineares, cujo tratamento matemático é mais amigável. Entretanto, essa metodologia não é mais sustentada numa era em que os recursos computacionais permitem estimar os parâmetros sem nenhuma manipulação ou mudança de variáveis do modelo original. O objetivo deste estudo é avaliar o erro na predição dos parâmetros cinéticos e no volume de reatores CSTR e PFR para uma reação do tipo $A \rightarrow B$, comparando o uso de dados simulados e parâmetros estimados através do método diferencial de análise para diversas ordens de reação.
\end{abstract}

\section{INTRODUÇÃO}

Os procedimentos de estimação de parâmetros, de forma geral, constituem a ponte que conecta as observações experimentais à interpretação teórica e quantitativa do problema. Estes são as ferramentas que tornam possíveis, dentre outras coisas, a simulação e o projeto de processos. Esta técnica consiste fundamentalmente em inferir os valores dos parâmetros que não podem ser medidos nem avaliados a priori, a partir de uma comparação estabelecida entre dados experimentais e um modelo disponível para o processo, cujo desempenho é afetado pelo parâmetro de interesse. (Schwaab e Pinto, 2007)

Para a cinética química, estes procedimentos são utilizados tendo em vista a identificação da lei de velocidade de uma determinada reação. Um dos tipos de reatores mais comuns para a obtenção de dados cinéticos é o reator em batelada. (Fogler, 2009) 


\subsection{Análise de Dados Cinéticos}

O estudo cinético é usualmente feito a partir de dados experimentais coletados durante a evolução de uma reação química. Este estudo é realizado, no caso do reator em batelada, através da medição da concentração em função do tempo, utilizando-se, a seguir, algum dos métodos de análise para os dados coletados, a fim de se determinar a ordem de reação e a constante de velocidade de reação. Quando uma reação é irreversível e conduzida em condições que permitam que a lei de velocidade seja uma função da concentração de apenas um dos reagentes, o método diferencial de análise pode ser aplicado. (Fogler, 2009)

\subsection{Método Diferencial de Análise}

Método de tratamento de dados utilizado para estimar as ordens de reação e os valores das constantes de velocidade de reação presentes na lei de velocidade. Para a reação $A \rightarrow B$ avaliada, o modelo cinético é: $-\frac{d C_{A}}{d t}=k C_{A}{ }^{\alpha}$, sendo k e $\alpha$ os parâmetros a determinar. $\mathrm{O}$ método diferencial propõe uma mudança de variáveis do modelo, de forma que este assume a forma linear $y=a x+b$, com $y=\ln \left(-\frac{d C a}{d t}\right)=\alpha \ln C_{A}+\ln k$. Pode-se ver que o método diferencial não avalia a variação temporal da concentração, mas a do logaritmo natural. Além disso, essa metodologia também necessita da avaliação numérica da derivada temporal da concentração, o que resulta em perda de precisão. Estes parâmetros são determinados na diferenciação numérica. As fórmulas de diferenciação de três pontos: (Fogler, 2009)

$$
\begin{aligned}
& \left(\frac{d C_{A}}{d t}\right)_{\mathrm{t}_{0}}=\frac{-\left.3 C_{A}\right|_{t_{0}}+\left.4 C_{A}\right|_{t_{1}}-\left.C_{A}\right|_{t_{2}}}{t_{2}-t_{0}} \\
& \left(\frac{d C_{A}}{d t}\right)_{\mathrm{t}_{0}<t_{n}<t_{N}}=\frac{\left.C_{A}\right|_{t_{n+1}}-\left.C_{A}\right|_{t_{n-1}}}{t_{n+1}-t_{n-1}} \\
& \left(\frac{d C_{A}}{d t}\right)_{\mathrm{t}_{N}}=\frac{\left.C_{A}\right|_{t_{N-2}}-\left.4 C_{A}\right|_{t_{N-1}}+\left.3 C_{A}\right|_{t_{N}}}{t_{N}-t_{N-2}}
\end{aligned}
$$

As equações (1) e (2) são utilizadas para calcular as derivadas no primeiro ponto e no último ponto, respectivamente, enquanto a equação (3) é usada para calcular a derivada em todos os pontos internos. (Fogler, 2009)

\section{MATERIAIS E MÉTODOS}

O trabalho consistem em avaliar o erro provocado pela utilização do método diferencial para estimar os parâmetros cinéticos para a reação elementar $A \rightarrow B$. Os dados simulados da concentração de reagente foram gerados com valor fixo de $\mathrm{k}$ e ordem entre $0 \mathrm{e}$ 2. Para tal, foi utilizado o softwere MATLAB para a simulação e estimação de parâmetros. 
Pela combinação do balanço molar com a lei de velocidade para uma reação elementar, temos (Fogler, 2009).

$-\frac{d C_{A}}{d t}=k C_{A}{ }^{n}$

$\mathrm{A}$ equação diferencial descrita pela $\mathrm{EQ} 4$ é resolvida para a condição inicial $\mathrm{C}_{\mathrm{A}}=\mathrm{C}_{\mathrm{A} 0}$ em $\mathrm{t}=0$, resultando na EQ5 (Coker, 2001).

$$
C_{A}=\left[k t(1-n)+C_{A 0}^{(1-n)}\right]^{\frac{1}{(1-n)}}
$$

A EQ5 foi utilizada para simular os dados da concentração do reagente $\mathrm{A} C a$ ao longo do tempo $t$. Adotou-se como concentração inicial de A $10 \mathrm{~mol} / \mathrm{L}$, velocidade específica da reação $k$ como 5 tempo $^{-1}\left(\frac{m o l}{L}\right)^{1-n}$ e o tempo variando de 0 a 1 unidades de tempo com passo de 0.1 . A ordem de reação $n$ foi variada de 0.05 a 2 no passo de tempo de 0.05 .

Para não haver uma indeterminação nos valores da matriz $\mathrm{Ca}$ nos pontos em que a ordem de reação é igual a 1, a EQ5 foi reescrita para uma reação de primeira ordem:

$$
C_{A}=C_{A 0} e^{-k t}
$$

Após a obtenção destes dados, o próximo passo foi aplicar o método diferencial de análise, a fim de estimar os parâmetros $n$ e $k$ da reação, para que estes sejam comparados com os dados simulados. Aplicando o logaritmo natural em ambos os membros da EQ4, temos:

$$
\ln \left(-\frac{d C_{A}}{d t}\right)=\ln k+n \ln C_{A}
$$

A aplicação do método diferencial resulta em uma relação linear entre $\ln (-d C a / d t)$ e $(\ln C a)$, cuja inclinação da reta é a ordem de reação $n$. Para obter a derivada $-d C a / d t$, foram aplicadas as equações EQ1 a EQ3. Feito isto, foi realizada a regressão linear, utilizando a ferramenta regress do software Matlab. Com os parâmetros da reação estimados a partir do método diferencial, fez-se a avaliação do erro percentual entre os parâmetros cinéticos (ordem de reação e velocidade específica) utilizados para gerar dados simulados e os parâmetros estimados, utilizando a EQ8.

$$
\frac{\text { real-experimental }}{\text { real }} \mid x 100
$$

Por fim, os parâmetros cinéticos utilizados para simulação e os valores estimados foram utilizados para a avaliação do erro na predição do volume dos reatores CSTR e PFR, cujas equações de projeto são dadas pelas EQ 9 e EQ 10, respectivamente (Fogler, 2009). 
Como referência, foi adotado um nível de conversão $(\mathrm{X})$ de $95 \%$, vazão volumétrica $\left(\mathrm{v}_{0}\right)$ de $10 \mathrm{~L} / \mathrm{s}$ e vazão molar de alimentação $\left(\mathrm{F}_{\mathrm{A} 0}\right) 100 \mathrm{mols} / \mathrm{h}$.

$$
\begin{aligned}
& V=\frac{C_{A 0} v_{0} X}{-k C_{A}{ }^{n}} \\
& V=F_{A 0} \int_{0}^{X} \frac{d X}{k C_{A}{ }^{n}}
\end{aligned}
$$

\section{RESULTADOS E DISCUSSÃO}

A partir da Figura 1 pode-se obervar que o erro percentual para a ordem de reação é relativamente baixo, atingindo $3 \%$ para a ordem de reação 2 . De modo semelhante, o erro percentual em relação à velocidade específica de reação também é pequeno, não chegando a 4\%. Embora esses valores possam ser considerados baixos, essa cenário pode não ser tão desprezível quando estamos tratando de estimação de dados cinéticos, uma vez que tais valores ditam diretamente a velocidade de reação e erros da ordem de $4 \%$ podem resultar em erros de projetos consideráveis quando se pensa na operação de um reator a longo prazo, o que é normal em reações ao nível industrial.

Assim, fica claro que deve haver cautela quanto ao uso dos métodos tradicionais de estimação de parâmetros, amplamente difundidos por sua simplicidade de aplicação, mas que, diante dos recursos computacionais hoje disponíveis, não se justifica mais tal aplicação.

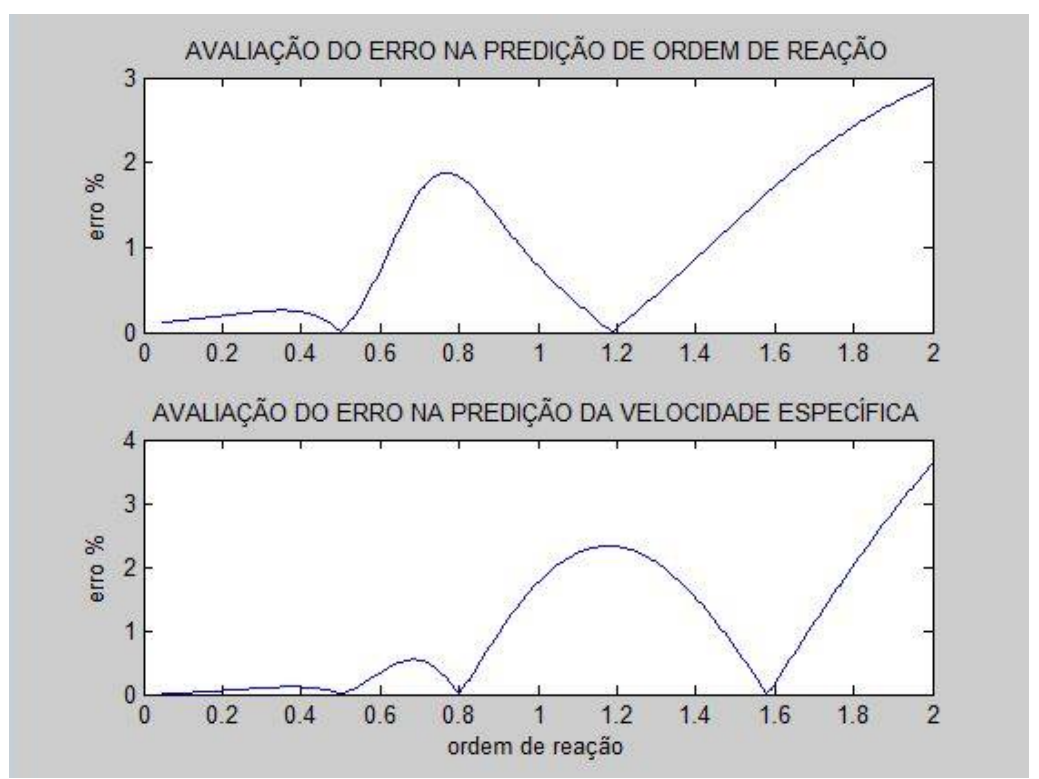

Figura 1: Avaliação do erro da predição de ordem de reação e avaliação do erro na predição da velocidade específica

As Figuras 2 e 3 apresentam as consequências desses erros na estimação dos parâmetros cinéticos sobre o projeto de reatores contínuos do tipo CSTR e PFR, respectivamente. São apresentados os desvios percentuais, em valores absolutos, para o volume do reator projetado com parâmetros cinéticos "reais" e aqueles estimados utilizando o 


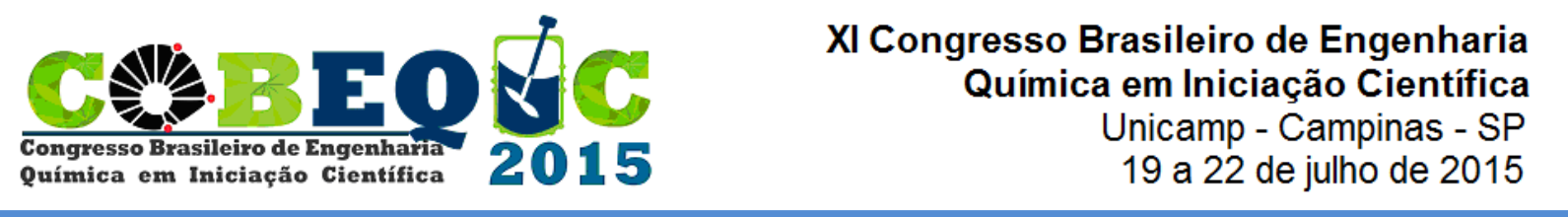

método dieferencial. Observa-se que para o reator PFR o erro é superior aos erros para o projeto do reator. Esse resultados lançam luz a uma questão importante no meio industrial: perdas. Se pensarmos, por exemplo, em um erro de projeto de $5 \%$ no volume do reator, temos dois cenários extremos: o subdimensionamento do reator, que resulta em conversão menor de reagente, o que resulta em menor capacidade de produção. Por outro lado, se pensarmos em um superdimensionamento do reator pode-se correr o risco desse erro resultar em alterações de tempo de residência que levem a formação de subprodutos ou até mesmo produtos indesejados e, no caso de um processo envolvendo um leito recheado, a subutilização do catalisador. Tais exemplos são apenas uma parte dos prejuízos que podem ocorrer a longo prazo em razão de erros aparentemente sutis no projeto de um reator químico.

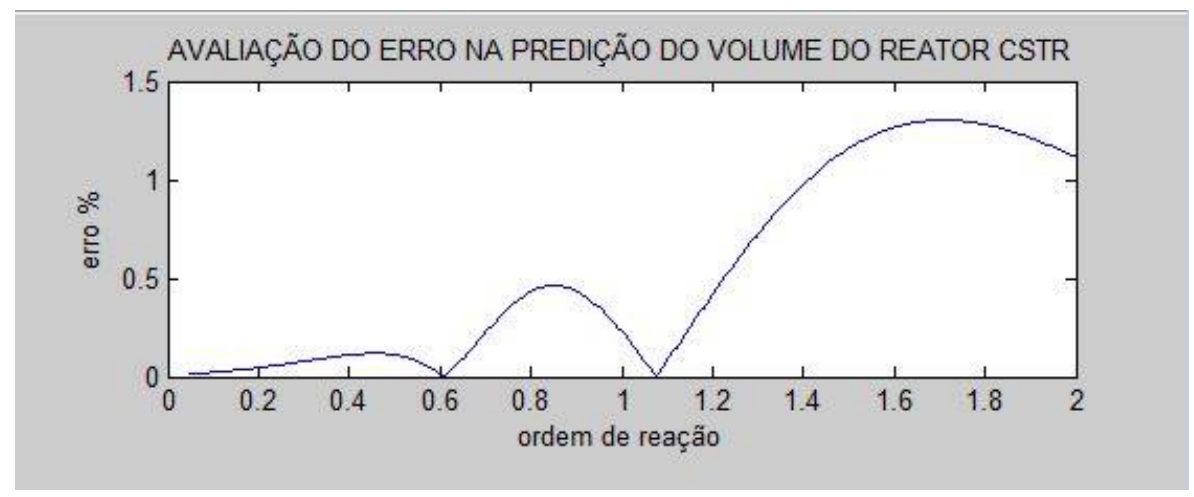

Figura 2: Avaliação do erro na predição do volume do reator CSTR

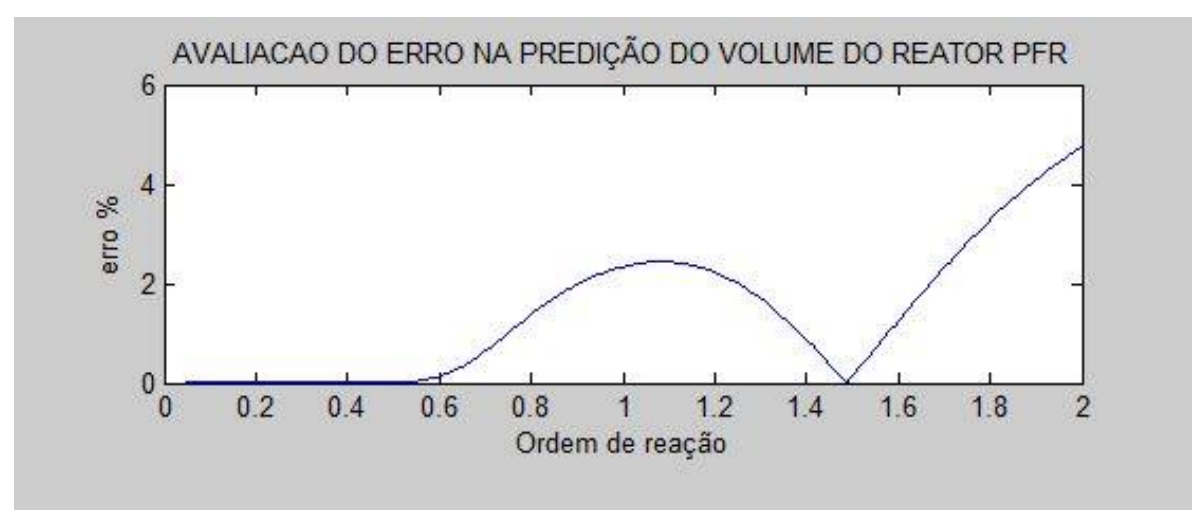

Figura 3: Avaliação do erro na predição do volume do reator PFR

\section{CONCLUSÕES}

O método diferencial de estimação de parâmetros cinéticos envolve mudanças de variáveis que tornam o processo de estimação mais fácil e prático para aqueles não tão familiarizados com as técnicas modernas de estimação de parâmetros. Entretanto, pode-se observar que tal prática deve ser adotada com prudência, uma vez que os resultados aqui apresentados mostram claramente que esse procedimento resulta em erros consideráveis, embora não o sejam para a maioria dos pesquisadores que utilizam essas ferramentas de estimação de parâmetros cinéticos. 
Em razão disso, o projeto de reatores químicos pode ficar comprometido, uma vez que ele necessita diretamente dos parâmetros cinéticos para o dimensionamento do reator, o que pode resultar numa série de problemas, como o super ou subdimensionamento, como problemas diretos e indiretamente pode resultar na mudança do tempo de residência, o que pode acarretar na formação de produtos que não são desejados ou até mesmo na utilização ineficiente de catalisador em processos nos quais eles se fazem necessários.

Nesse sentido, fica claro que deve haver prudência na utilização de tais métodos, ao mesmo tempo que deve-se incentivar o uso de ferramentas mais sofisticadas na estimação de parâmetros, uma vez os recursos computacionais e numéricos estão cada vez mais disponíveis.

\section{REFERÊNCIAS}

COKER, A. K. Modeling of Chemichal Kinetics and Reactor Design. Houston: Editora Gulf Publishing Company, 2001.

FOGLER, H. S. Elementos de Engenharia das Reações Químicas. 4.ed. Rio de Janeiro: Editora LTC, 853 p., 2009.

SCHWAAB, M.; PINTO, J. C. Análise de Dados experimentais I - Fundamentos de Estatística e Estimação de Parâmetros. Rio de Janeiro: Editora E-papers, 2007. 